OPEN ACCESS

Edited by:

Andrea Cavani,

UOC Coordinamento

Scientifico_National Institute for Health

Migration and Poverty (NIHMP), Italy

Reviewed by:

Stefania Madonna

Istituto di Dermatologia Immacolata

(IRCCS), Italy

Elena Donetti,

University of Milan, Italy

*Correspondence:

Erik Lubberts

E.Lubberts@erasmusmc.n

Specialty section:

This article was submitted to

Cytokines and Soluble

Mediators in Immunity,

a section of the journal

Frontiers in Immunology

Received: 03 June 2021

Accepted: 25 August 2021

Published: 20 September 2021

Citation:

$X u X$, Prens E, Florencia E, Leenen $P$, Boon L, Asmawidjaja P, Mus A-M and Lubberts $E$ (2021) Interleukin-17A

Drives IL-19 and IL-24 Expression in

Skin Stromal Cells Regulating Keratinocyte Proliferation.

Front. Immunol. 12:719562. doi: 10.3389/fimmu.2021.719562

\section{Interleukin-17A Drives IL-19 and IL- 24 Expression in Skin Stromal Cells Regulating Keratinocyte Proliferation}

\author{
Xiaofei $\mathrm{Xu}^{1,2,3}$, Errol Prens $^{2}$, Edwin Florencia ${ }^{1,2,3}$, Pieter Leenen $^{3}$, Luis Boon ${ }^{4}$, \\ Patrick Asmawidjaja ${ }^{1,3}$, Anne-Marie Mus ${ }^{1,3}$ and Erik Lubberts ${ }^{1,3^{*}}$ \\ ${ }^{1}$ Department of Rheumatology, Erasmus Medical Center, Rotterdam, Netherlands, ${ }^{2}$ Department of Dermatology, Erasmus \\ Medical Center, Rotterdam, Netherlands, ${ }^{3}$ Department of Immunology, Erasmus Medical Center, Rotterdam, Netherlands, \\ ${ }_{4}^{4}$ Polypharma Biologics, Utrecht, Netherlands
}

IL-17A has been shown to be up-regulated in psoriasis lesions and is central to psoriasis pathogenesis. IL-19, along with other IL-20 subfamily cytokines such as IL-20 and IL-24, is induced by IL-17A and contributes especially to epidermal hyperplasia in psoriasis. However, the regulation, cellular sources of IL-19 and whether targeting of IL-17A by biologics influence IL-19 expression is not completely understood. To investigate the regulation of IL-19 by IL-17A in psoriasis, the imiquimod-induced psoriasis mouse (IMQ) model was used. Enhanced expression of IL-17A in the IMQ model was achieved by antiIL-10 antibody treatment. Assessments of skin inflammation macroscopically, by histology and flow cytometry, all confirmed increased psoriatic symptoms. Interestingly, depletion of IL-10 markedly upregulated IL-23/IL-17 pathway related cytokines followed by a significant increase in IL-19 and IL-24. The up-regulation of IL-19 and IL-24, but not IL-17A, coincided with increased keratinocyte proliferation. To investigate the cellular source and effects of biologics on IL-19, human skin fibroblasts from healthy controls and psoriasis patients were cultured alone or co-cultured with activated memory CD4+ T cells. Besides IL-1 $\beta$, IL-17A induced direct expression of IL-19 and IL-24 in skin fibroblasts and keratinocytes. Importantly, intrinsic higher expression of IL-19 in psoriatic skin fibroblasts was observed in comparison to healthy skin fibroblasts. Neutralization of IL-17A in the human skin fibroblast-T cell co-culture system significantly suppressed IL-19 and IL-24 expression. Together, our data show that IL-17A-induced IL-19 and IL-24 expression in skin stromal cells contribute to keratinocyte proliferation.

Keywords: psoriasis, cytokines, skin, inflammation, IL-17A, Th17

\section{INTRODUCTION}

Psoriasis is a chronic autoimmune skin disease affecting around 2 to $3 \%$ of the Western population $(1,2)$. Psoriasis vulgaris, the plaque-forming phenotype, is the most common type and accounts for $85-90 \%$ of all patients with psoriasis $(1,3)$.

Aberrant regulation of pro-inflammatory and anti-inflammatory cytokines is considered important in the pathogenesis of psoriasis (1-4). Increased levels of IL-17A and elevated percentages of IL-17A-producing lymphocytes are found in psoriatic plaques (5-9). IL-17A alone, and in combination with other inflammatory cytokines such as TNF $\alpha$, stimulate 
keratinocyte activation, proliferation, amplifies the immune response and perpetuates cell infiltration (10, 11). Biologics targeting IL-17A alone or in combination with IL-17F or the IL-17 receptor A (IL-17RA) are efficacious in the treatment of plaque psoriasis, highlighting the central role of the IL-17A pathway in psoriasis pathogenesis (12). Interleukin-19, a member of the IL-20 subfamily together with IL-20 and IL-24, is up-regulated in psoriatic lesions and contributes to keratinocytes hyperplasia in psoriasis (13). IL-19 is reportedly down-stream of the IL-23/IL-17 cascade and autocrine production of IL-19 by keratinocytes is shown to elicit keratinocyte proliferation (14). Recent data show that serum IL-19 levels reflect clinical improvement induced by anti-IL-17 biologic treatment (15). However, whether other resident cells, such as skin fibroblasts, are a source of IL-19 and whether in situ IL-19 expression is normalized in patients with psoriasis treated with anti-IL-17 therapy is not clear.

Previously, our group established a psoriasis-like skin inflammation model in mice using topical application of imiquimod (IMQ) (16). This model successfully re-captures most critical features of acute plaque formation in psoriasis such as keratinocyte hyper proliferation, acanthosis and parakeratosis (16). Like in human psoriasis, enhanced activity of the IL-23/IL-17 pathway was also involved in the IMQ-induced psoriasis mouse model (16). However, in contrast to the chronic natural course in human psoriasis, this mouse model does not develop into a chronic state of psoriasis, because of stabilization and even improvement of skin inflammation after 5 to 6 days. Interestingly, a clinical study in psoriasis patients showed that, non-lesional skin treated with IMQ initially developed typical features of psoriasis such as acanthosis and parakeratosis (17). Nevertheless, both clinical and histological features subsided thereafter and in this human model of IMQ-induced psoriasis, the induced lesions showed spontaneous improvement after 5 to 6 days. This improvement was accompanied by significantly lower expression of IL-17A and with a higher expression of IL-10 (17). This suggests that upregulation of IL-10 is involved in the spontaneous improvement of psoriasis symptoms after 5 to 6 days in murine IMQ model and probably explains the spontaneous improvement observed in the IMQ mouse model. Therefore, we used an anti-IL-10 antibody to investigate whether we could achieve enhanced expression of IL-17 in the IMQinduced psoriasis mouse model and the accompanying visible psoriatic symptoms beyond day 5. In vitro assays with human skin fibroblasts from patients with psoriasis and healthy skin were performed to evaluate the direct induction of IL-19 by IL-17. In addition, an ex vivo human psoriasis skin co-culture system was used to examine the effects of biologics targeting IL-17A on IL19 expression.

\section{MATERIAL AND METHODS}

\section{IMQ-Induced Psoriasis Mouse Model}

$\mathrm{BALB} / \mathrm{c}$ mice (8-11 week-old) received daily topical application of $62.5 \mathrm{mg} 5 \%$ Aldara (3M Pharmaceuticals) on their shaved back skin. Control mice $(n=6$, pooled from two independent experiments) were treated with a thin layer of petrolatum (Fagron). Daily evaluation of the local psoriasis area and severity index (PASI) has been described previously (16). Every other day, $20 \mathrm{mg} / \mathrm{kg}$ body weight of anti-IL-10 or isotype control antibody $(\mathrm{n}=10$ each, pooled from two independent experiments) was intraperitoneally (i.p.) injected, or $5 \mathrm{mg} / \mathrm{kg}$ body weight of dexamethasone $(n=7$, pooled from two independent experiments) was subcutaneously (s.c.) injected as an anti-inflammatory gold standard. Five and ten days after IMQ induction, mice were sacrificed for analysis. Food and water were provided ad libitum, and mice were kept under specific pathogen-free conditions. All experiments were approved by the Erasmus MC Dutch Animal Ethics Committee (DEC).

\section{Histology and Immunohistochemistry}

After sacrifice, skin biopsies were taken and snap-frozen in TissueTek (Bayer). Sections were cut with a Leica cryostat. Gr1 antibody (clone RB6-8C5) and Ki-67 antibody (Dako, A0047) were used for IHC staining. Subsequent steps were performed as described earlier (16).

Images were analyzed with LAX V4.12 program (Leica microsystems) or NDP view2 (Hamamatsu photonics). To measure epidermal thickness, the average of four measurements was used as the representative thickness per sample. To reduce variance between different experiments, thickness ratios were calculated. Specifically, each skin thickness was divided by the mean skin thickness of the isotype group from that experiment, and thereby mean values of thickness for isotype groups were always set at one.

\section{Flow Cytometry}

Back skin (ca. $1 \mathrm{~cm} 2$ ) was digested in $50 \mu \mathrm{g} / \mathrm{mL}$ Liberase (Roche) at $4^{\circ} \mathrm{C}$ overnight and then at $37^{\circ} \mathrm{C}$ for 1 hour to create a single cell suspension and cells were stained with the following antibodies: CD45-BV785 (Biolegend, clone 104), CD11b-eF450 (eBioscience, clone M1/70), Ly6C-APC-Cy7 (BD pharmingen, clone AL-21) and Ly6G-PE-CF594 (BD horizon, clone 1A8). Samples were analyzed with LSR II flow cytometer (BD Biosciences) and results processed with FlowJo software (TreeStar).

Healthy peripheral blood mononuclear cells (PBMC) were obtained from buffy coats (Sanquin, Amsterdam, the Netherlands) and harvested with Ficoll density gradient centrifugation. For the co-culture experiments, memory $\mathrm{T}$ cells (CD4+CD45RO+CD14-CD25low/int) were sorted using the FACSAria cell sorter (BD Biosciences) and co-cultured with skin fibroblasts. Cells were stained with the following antibodies: CD14-APC-H7 (clone MфP9), CD45RO-PerCPCy5.5 (clone UCHL1), both from BD Biosciences, and CD4FITC (clone RPA-T4), CD25-PE Cy7 (clone BC96), both from Sony Biotechnology. The obtained cell purity was $\geq 98 \%$.

\section{Reverse Transcription and Real-Time Polymerase Chain Reaction}

RNA was isolated with TRIzol (Thermo Fisher) or Total RNA Miniprep Kit (Sigma-Aldrich). cDNA was synthesized with Superscript II after DNase treatment (both from Invitrogen). RT-PCR was performed with ViiA7 sequence detection system 
(Life Technologies). Gene expression of IL-17A, IL-17F, IL-19, IL-22, IL-23p19, IL-24 were normalized to glyceraldehyde 3phosphate dehydrogenase $(\mathrm{GAPDH})$ in mouse samples and hypoxanthine-guanine phosphoribosyltransferase (HPRT) in human samples. Primer sequences were summarized in Supplementary Table S1.

\section{Human Primary Skin Fibroblast Cultures and Co-Cultures With T Cells}

Fibroblasts $(n=6)$ from lesional skin of psoriasis patients were obtained from our biobank collection. Healthy skin samples $(n=6)$ were obtained from healthy individuals who underwent cosmetic surgeries in the Sint Franciscus Hospital (Rotterdam, The Netherlands). Signed consents were provided by all healthy participants. Human primary skin fibroblasts were cultured from above skin samples as described previously (18). Passages 3-8 fibroblasts were seeded $1.0 \times 10^{4}$ per well in 96-well culture plates and stimulated with IL-1 $\beta$ (0.01 ng/mL, 201-LB), TNF $\alpha$ (5 ng/ $\mathrm{mL}, 210-\mathrm{TA}$ ), IL-17A (50 ng/mL, 317-IL), and IL-17F (500 ng/ $\mathrm{mL}, 1335-\mathrm{IL}$ ) (all from R\&D systems) for 24 hours (hrs).

In co-culture experiments, skin fibroblasts $\left(1.0 \times 10^{4}\right)$ were cocultured with $\mathrm{CD} 4+\mathrm{CD} 45 \mathrm{RO}+\mathrm{CD} 14-\mathrm{CD} 25$ low/int $\mathrm{T}$ memory cells $\left(2.5 \times 10^{4}\right)$ sorted from buffy coats $(n=6)$. Soluble anti-CD3 and anti-CD28 (both from Sanquin, Amsterdam, The Netherlands) were added for 72 hrs. In addition, $100 \mu \mathrm{g} / \mathrm{ml}$ anti-IL-17A antibody (secukinumab, Novartis), $1 \mu \mathrm{g} / \mathrm{ml}$ antiTNF antibody (adalimumab, AbbVie), and an isotype IgG1 antibody (Sigma-Aldrich) were used.

\section{Enzyme-Linked Immunosorbent Assay}

Human IL-8 and IL-19 in culture supernatants was measured with ELISA (Invitrogen) and ELISA Duoset (R\&D systems) following manufacturers' instructions.

\section{Data Set Analysis}

Public microarray data (GSE13355) were analyzed to compare mRNA expression of psoriatic lesional $(n=64)$, non-lesional skin $(n=58)$ and skin from healthy controls $(n=58)$. Based on GSE13355, values of 216876_s_at (IL-17A), 220745_at (IL-19), 206569_at (IL-24) and 212021_s_at (MKI67) from gene expression profile GDS4602 were plotted. Dataset GSE53552 was analyzed for mRNA expression of psoriatic skin lesions following treatment with brodalumab (AstraZeneca). Psoriatic non-lesional $(n=23)$, lesional $(n=25)$, and day $8(n=4)$, day 15 $(\mathrm{n}=19)$, day $43(\mathrm{n}=16)$ after treatments were included. 220745_at (IL-19), 206569_at (IL-24), 212022_s_at (MKI67) values from GDS5420 were plotted based on GSE53552.

\section{Statistics}

Statistical differences were determined with paired or unpaired student's $t$ test. All data analyses were performed with GraphPad Prism V5 and P-values $<0.05$ were considered as significant.

\section{RESULTS}

\section{IL-10 Neutralization Enhances Skin Thickness and Scaling in the IMQ-Induced Psoriasis Mouse Model}

The design of the IL-10 neutralization experiments is summarized in Supplementary Figures S1A, B. Local psoriasis area and severity index (PASI) score was used to evaluate psoriasis symptoms including skin scaling, thickness and redness. As shown in Figure 1A, ten days after IMQ treatment, macroscopic scores of skin scaling and thickness were significantly higher in the anti-IL-10 treated group (anti-IL-10) compared to the isotype antibody control group (isotype). This resulted in a significant higher PASI score after neutralizing IL-10 compared to the isotype control (Figure 1A). Dexamethasone treatment significantly
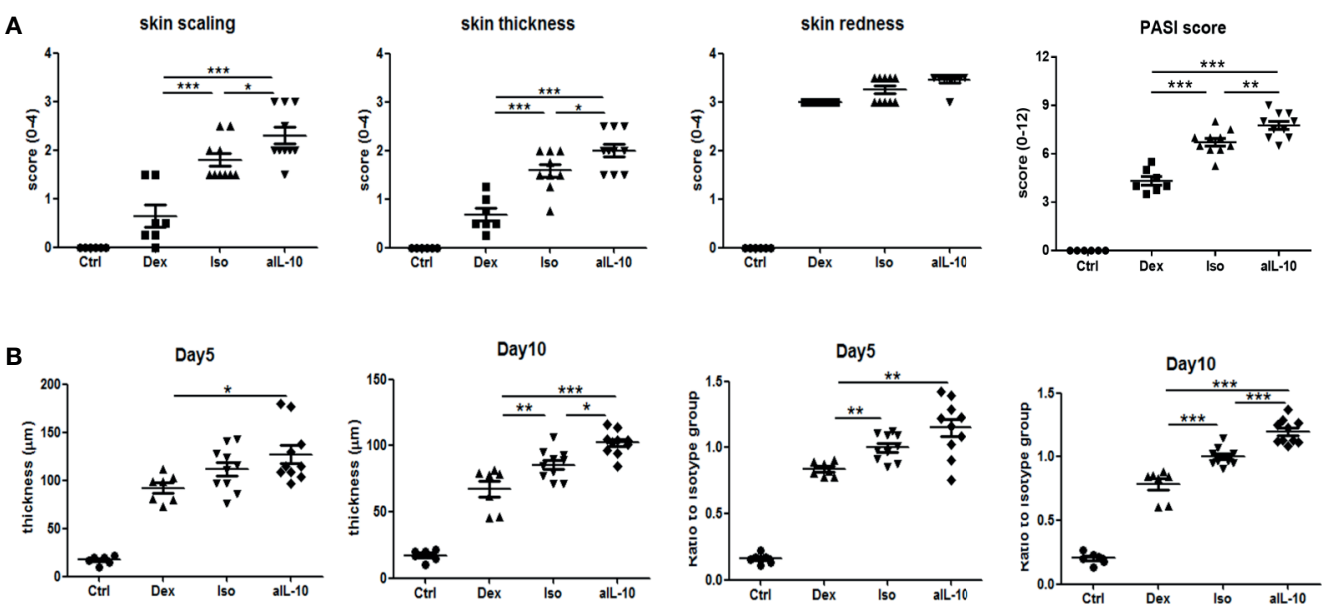

FIGURE 1 | IL-10 neutralization worsens psoriatic symptoms and epidermal thickness in the IMQ-induced psoriasis mouse model. (A) At day 10, scores for skin scaling, thickness, redness and PASI in various groups following IMQ treatment. (B) Measured average epidermal thickness in psoriasis-like skin at days 5 and 10 , and thickness ratios compared to isotype antibody controls at days 5 and 10 . Data are shown as means \pm SEMs. ${ }^{\star} P<0.05$, ${ }^{\star \star} P<0.01$, and ${ }^{\star \star \star} P<0.001$. 
improved both symptoms compared to either the anti-IL-10 or isotype group. No significant difference for skin redness was observed among groups. Details of kinetic data of the macroscopic scores were summarized in Supplementary Figure S2A. These data indicate that IL-10 neutralization enhances skin thickness and scaling in the IMQ-induced psoriasis mouse model beyond day 5 .

\section{IL-10 Neutralization Increases Epidermal Thickness and Keratinocyte Proliferation in the IMQ-Induced Psoriasis Mouse Model}

To confirm the increased skin thickness observed macroscopically after anti-IL-10 treatment, H\&E staining was performed and epidermal thickness was measured microscopically (Figure 2A). At day 5, epidermal thickness was significantly higher in the antiIL10 compared to dexamethasone treatment (Figure 1B). At day 10, anti-IL-10 significantly increased epidermal thickness compared to the isotype group, whereas dexamethasone significantly reduced this compared to both groups (Figure 1B). This is in line with our macroscopic findings of increased skin thickness with the PASI score in the anti-IL-10 treated group (Figure 1A).

As indicated before, compared to isotype group, ratios of epidermal thickness in the anti-IL-10 treated group were slightly increased at day 5, but significantly increased at day 10 (Figure 1B). Both epidermal thickness and thickness ratios in the isotype group were comparable to saline-treated IMQ groups (Supplementary Figure S2B). Ki-67 staining was performed to further identify proliferating cells. As shown in Figure 2B, 1-2 layers of keratinocytes in the epidermal stratum basale were Ki$67+$ in dexamethasone group, 2-3 layers were Ki-67+ in isotype group, while 4-5 layers were Ki-67+ in anti-IL-10 group (at days 5 and 10). This is in line with the increased thickness of the epidermis in the anti-IL-10 treated group at day 10 (Figure 1B). Taken together, our data indicate that in the IMQ-induced psoriasis mouse model, IL-10 neutralization enhances skin thickness through facilitating keratinocyte proliferation.

\section{IL-10 Neutralization Increases the Recruitment of Neutrophils and Monocytes Into the Skin}

Flow cytometry showed representative staining results of infiltrating neutrophils (CD11b+Ly6CintLy6G+) in lesional skin at day 10 (Figure 3A). Both neutrophil numbers and percentages were increased after neutralization of IL-10 compared to the isotype group (Figure 3B). Treatment with dexamethasone significantly reduced both parameters compared to the other groups (Figure 3B). At days 5 and 10, IHC staining confirmed the enhanced recruitment of Gr-1+ neutrophils in IMQ lesional skin in the anti-IL-10 treated group compared to the isotype group (Figure 3C). Neutrophil chemokine CXCL2, but not CXCL1, was upregulated after neutralizing IL-10 and correlated with enhanced neutrophil recruitment (Figure 3B). Similar results were found for CD11c+Ly6Cint monocytesderived dendritic cells (mono/DCs) (Figures 3D, E). The isotype group showed similar cell numbers and percentages of neutrophils and mono/DCs compared to the saline-treated IMQ

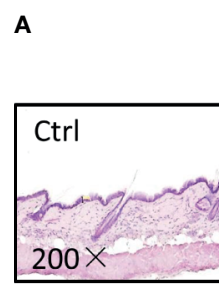

Day5
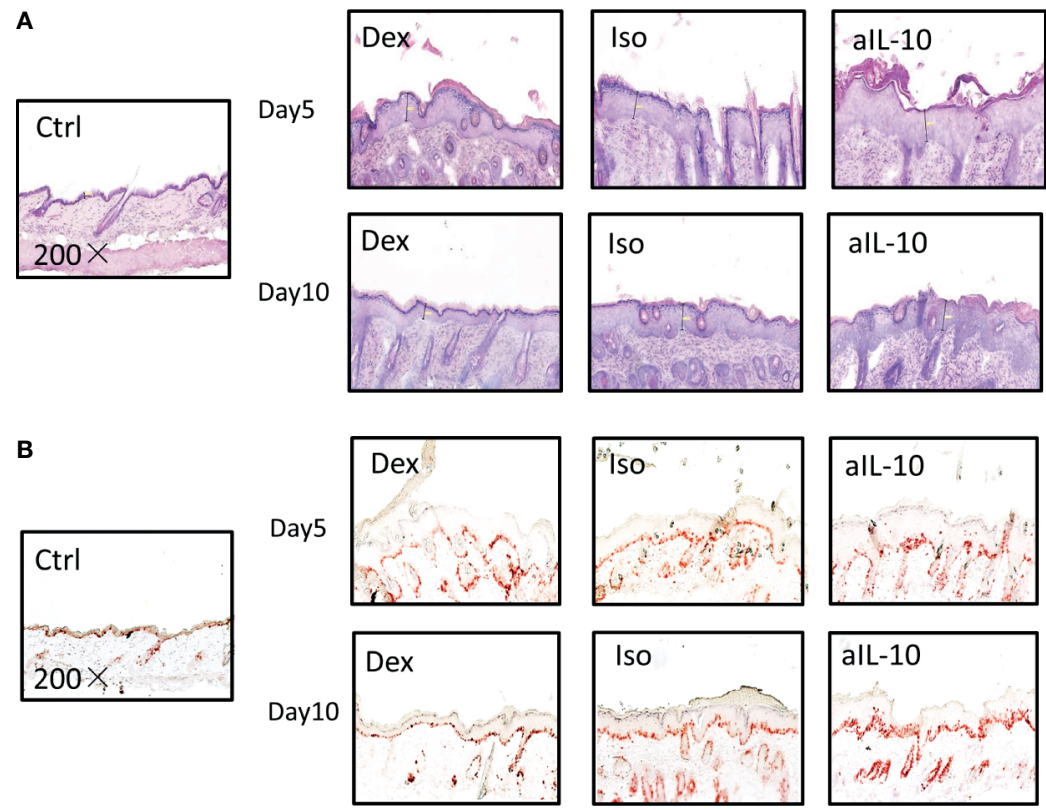

FIGURE 2 | IL-10 neutralization increases keratinocyte proliferation and neutrophil accumulation in the IMQ-induced psoriasis mouse model. (A) Representative H\&E staining results of skin sections in IMQ-induced psoriasis mouse model at days 5 and 10. (B) Representative $\mathrm{IHC}$ staining of Ki-67+ proliferating keratinocytes in skin sections of IMQ-induced psoriasis mouse model at days 5 and 10. All images were taken with 200× magnification. 
group (Supplementary Figures S2C, D), indicating that no specific immune effects were induced by the isotype antibody injections. These data indicate that IL-10 neutralization in the IMQ-induced psoriasis mouse model results in enhanced persistent inflammation, as evidenced by enhanced influx of neutrophils and mono/DCs into lesional skin.

\section{IL-10 Neutralization in the IMQ-Induced Psoriasis Mouse Model Results in an Early Upregulation of the IL-23/IL-17 Immune Pathway Related Cytokines Followed by a Subsequent Later Increase of IL-19 and IL-24 in the Skin}

In psoriasis, IL-17-producing T helper cells (Th17) are central in the pathogenesis and Th17-related cytokines such as IL-17A, IL$17 \mathrm{~F}$ and IL-22, together with TNF $\alpha$, drive epidermal hyperplasia $(9,16,19)$. Therefore, we investigated the effects of IL-10 neutralization on $\mathrm{T}$ cells and $\mathrm{T}$ cell cytokine expression in the IL-23/IL-17-dependent IMQ-induced psoriasis mouse model (16). Neutralizing IL-10 did not change the number of CD3+ $\mathrm{T}$ cells, including CD4+ and $\gamma \delta \mathrm{T}$ cells at day 10 (Supplementary
Figures S3A-C). However, at day 5, IL-23p19, IL-22, IL-17A and IL-17F were significantly increased in the anti-IL-10 treated group compared to the isotype control group (Figure 4A). Interestingly, no difference in expression of these cytokines was found between these two groups at day 10 (Figure 4A). In contrast, the IL-20 subfamily cytokines, IL-19 and IL-24, were significantly upregulated only at day 10 but not at day 5 (Figure 4B) which correlated with the significant increase of epidermal thickness at day 10. This indicates that IL-19 and IL24 , rather than IL-22, IL-17A or IL-17F, were responsible for the late stage (day 10) keratinocyte hyper-proliferation and acanthosis in the IMQ-induced psoriasis mouse model during anti-IL-10 treatment.

\section{IL-17 Induces Human Skin Fibroblasts to Produce IL-19 and IL-24}

As the increase in IL-17 family cytokines preceded the upregulation of IL-19 and IL-24, we assumed that the IL-17 family cytokines induced IL-19 and IL-24. Therefore, the expression of IL-19 and IL-24 in skin fibroblasts was examined after stimulation with IL-1 $\beta$, TNF, IL-17A, or IL-17F for 24

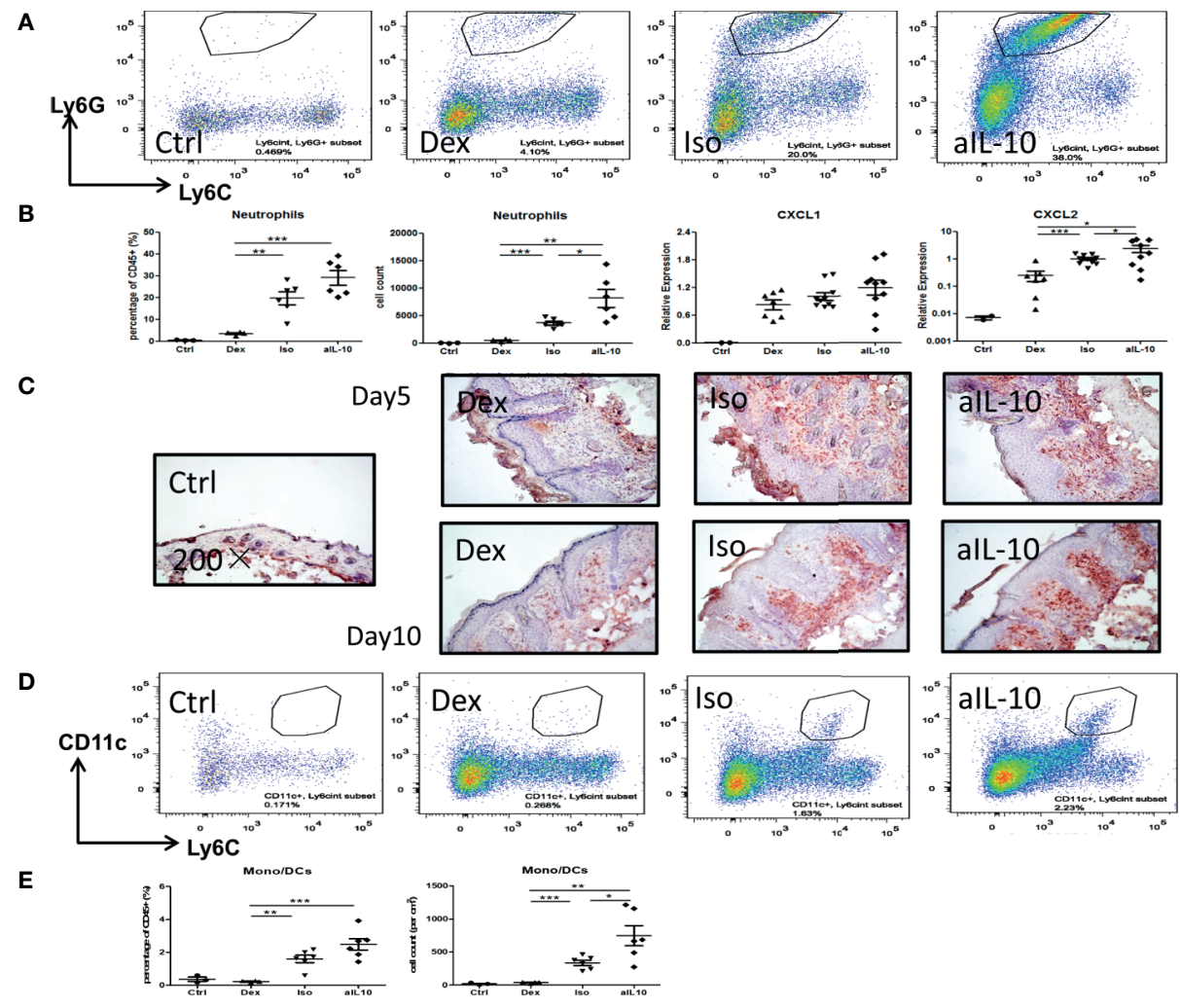

FIGURE 3 | IL-10 neutralization increases neutrophil and monocyte/DC infiltration in the IMQ-induced psoriasis mouse model. (A) Flow cytometry staining of Ly6G+ Ly6Cint neutrophils among pregated CD45+CD11b+ cells in lesional psoriasis-like skin at day10. (B) Percentages and cell numbers of neutrophils among total CD45+ immune cells at day 10 in flow cytometry analysis and levels of the neutrophil chemokines, CXCL1 and CXCL2, in lesional psoriasis-like skin. (C) Representative IHC staining of Gr-1+ neutrophils in skin sections of the IMQ-induced psoriasis mouse model at days 5 and 10. (D) Flow cytometry staining of CD11C+Ly6Cint monocytederived dendritic cells in lesional psoriasis-like skin at day10. (E) Percentages and cell numbers of monocyte-derived dendritic cells (mono/DCs) among total CD45+ immune cells at day 10 using flow cytometry. Data are shown as means \pm SEMs. ${ }^{\star} P<0.05,{ }^{\star \star} P<0.01$, and ${ }^{\star \star \star} P<0.001$. 
hours. The expression of the IL-17 receptor, IL-17RA and IL17RC, was confirmed on skin fibroblasts from psoriasis patients or healthy volunteers (Supplementary Figure S4A). As shown in Figure 5A, both IL-17A and IL- $1 \beta$ significantly increased IL-19 and IL-24 mRNA expression compared to the unstimulated group. TNF, on the other hand, was only a significant inducer of IL-24 (Figure 5A). Additionally, we observed an intrinsic higher expression of IL-19 and IL-24 in psoriatic skin fibroblasts compared to skin fibroblasts from healthy controls (Figure 5A).

To further explore the induction of IL-19 and IL-24 by IL$17 \mathrm{~A}$, healthy and psoriasis skin fibroblasts were co-cultured with CD4+CD45RO+CD14-CD25low/int T memory cells for 72 hours with or without stimulation. Neutralizing antibodies for IL-17A, TNF or the combination were added to these cocultures. Figure 5B showed that anti-IL-17A treatment significantly reduced mRNA expression of IL-19 and IL-24 compared to either the isotype antibody or anti-TNF. In contrast, anti-TNF treatment only significantly reduced expression of IL-19 but not of IL-24 (Figure 5B). No additive effect was shown when both IL-17A and TNF were neutralized compared to anti-IL-17 treatment alone. In line with the mRNA expression data, ELISA data showed that both anti-IL-17A and anti-TNF significantly reduced the protein levels of IL-19 in the co-culture supernatants with anti-IL-17 more potent than anti-TNF, but without additive effect when treatment was combined (Figure 5C). However, anti-IL17A and anti-TNF both significantly reduced the levels of IL-8, with additive effect when combined (Figure 5C). Also in these skin fibroblast-T cell co-cultures, IL-19 and IL-24 mRNA expression as well as protein levels of IL-19 and IL-8 were all lower when skin fibroblast were derived from healthy controls compared to patients with psoriasis (Figures 5B, C). This indicates an intrinsic higher expression of both cytokines in psoriasis lesions.

IL-17A-induced expression of IL-19 was also checked in healthy human skin primary keratinocytes. Expression of the IL-17 receptor, IL-17RA and IL-17RC, was confirmed in these keratinocytes (Supplementary Figure S4A). IL-17A stimulation of human keratinocytes resulted in increased IL-19 mRNA expression. On the other hand, inhibition of IL-17A in keratinocyte-T cell co-cultures reduced IL-19 mRNA expression (Supplementary Figure S4B). Overall, these data indicate that IL-17A induces IL-19 and IL-24 expression in human skin resident cells such as fibroblasts and keratinocytes, and IL-17A blockade reduces their expression.

\section{IL-17A Neutralization Reduces IL-19 and Cell Proliferation in Psoriatic Skin Lesions}

Gene expression data from psoriatic lesional skin, psoriatic nonlesional skin, and healthy skin, obtained in clinical trials with antiIL-17 biologics in psoriasis, were analyzed using the Genomic Spatial Event (GSE) database. As shown in Figure 6A, IL-17A, IL19, and IL-24 were all significantly higher in psoriatic lesional skin compared to either psoriatic non-lesional skin or healthy skin. This corroborated our findings of a higher expression of IL-19 and IL-24 in psoriatic skin fibroblasts compared to healthy fibroblasts

(Figure 5). Furthermore, Ki-67, was significantly increased in psoriatic lesional keratinocytes (Figure 6A). Significant
A Day5
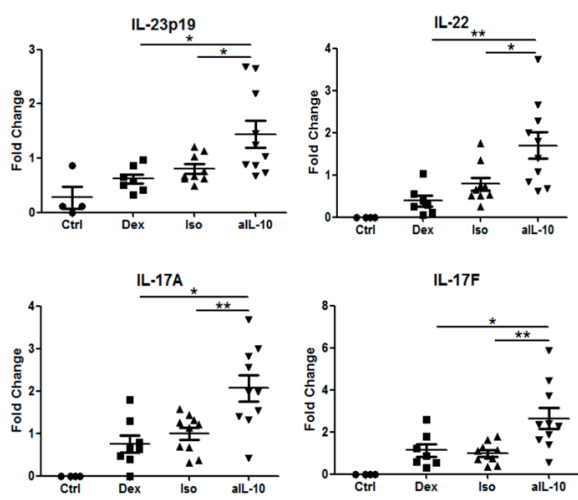

B Day5

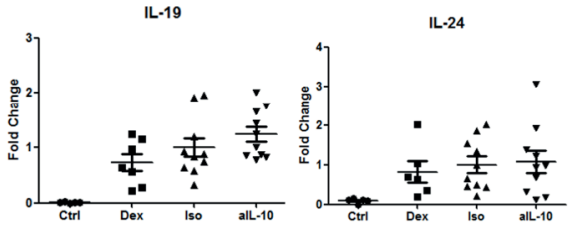

Day10
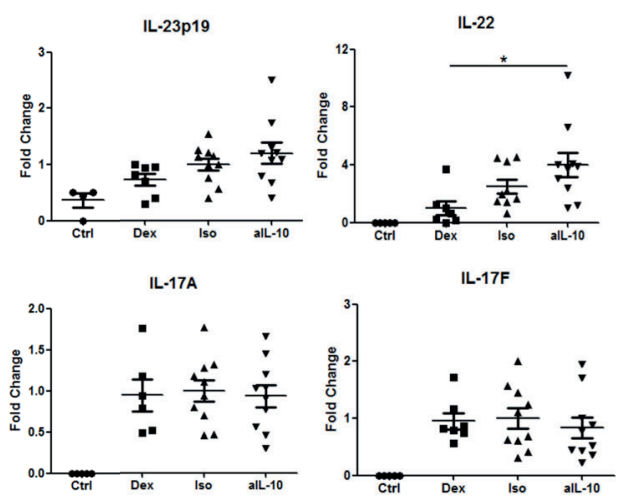

Day10
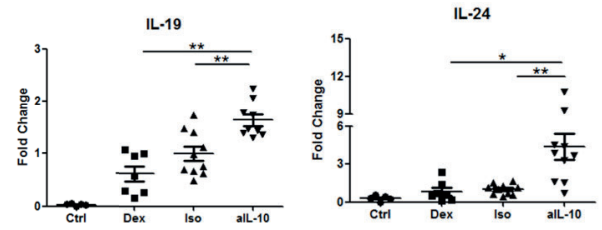

FIGURE 4 | Early and late up-regulation of IL-23/IL-17 cytokines and IL-20 subfamily cytokines in the skin during anti-IL-10 treatment in IMQ-induced psoriasis mouse model. (A) At days 5 and 10, mRNA expression of IL-23, IL-22, IL-17A and IL-17F in various groups following IMQ treatment. (B) At days 5 and $10, \mathrm{mRNA}$ expression of IL-19 and IL-24 in various groups following IMQ treatment. Data are shown as means \pm SEMs. ${ }^{*} P<0.05,{ }^{* *} P<0.01$. 
A

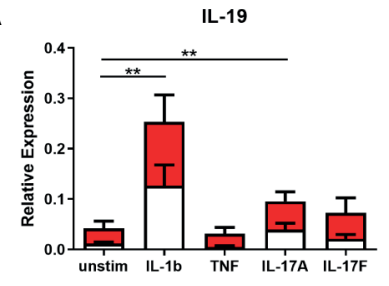

B

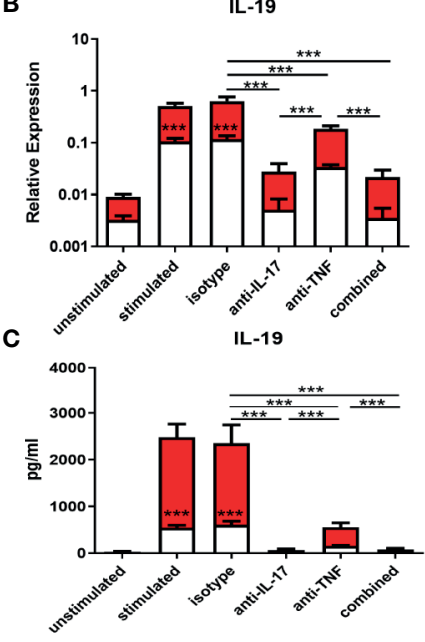

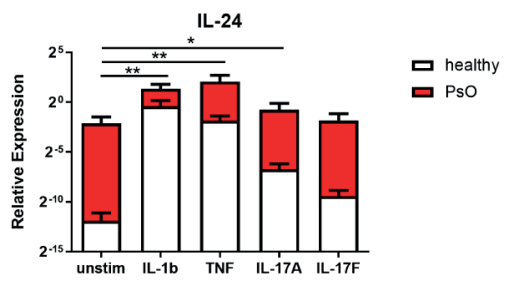

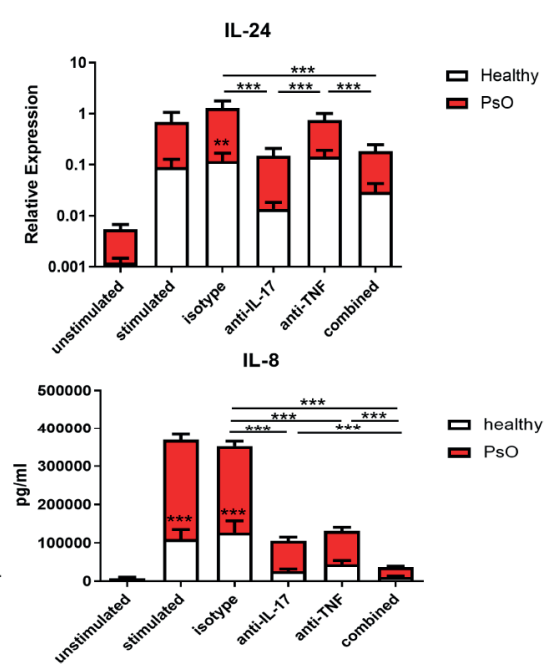

FIGURE 5 | IL-17A induces IL-19 and IL-24 expression in human skin resident cells such as fibroblasts and keratinocytes, and IL-17A blockade reduces their expression. (A) IL-19 and IL-24 mRNA expression in fibroblasts of healthy donors $(n=6)$ and psoriasis patients ( $\mathrm{n}=4$ ) without stimulation or stimulated with IL-1 $\beta$, TNF $\alpha$, IL-17A and IL-17F for 24 hours. (B) Healthy control skin fibroblasts and psoriatic fibroblasts (n=6 each) were co-cultured with unstimulated or anti-CD3/antiCD28 stimulated memory CD4+ T cells from healthy controls for 72 hours. IL-19 and IL-24 mRNA expression in these co-cultures without treatment or treated with an anti-IL-17A antibody, anti-TNF antibody, their combination or an isotype control antibody. (C) Levels of human IL-19 and IL-8 protein in the supernatant of the above mentioned co-cultures without treatment or treated with an anti-IL-17A antibody, anti-TNF antibody, their combination or an isotype control antibody. Data are shown as means \pm SEMs. ${ }^{\star} P<0.05,{ }^{\star *} P<0.01$, and ${ }^{* \star *} P<0.001$.

upregulation of IL-17A, IL-19, IL-24, and Ki-67 in psoriasis was confirmed in another independent dataset (Supplementary Figure S5A).

In a separate dataset, in which an IL-17 receptor A (IL-17RA) antibody was used in the treatment of psoriasis, a significant reduction of the expression of IL-19, IL-24, and Ki-67 in psoriatic lesional skin was found, reducing their expression levels close to those of non-lesional skin after treatment (Figure 6B). Similar significant down-regulation of IL-19 and Ki-67 was confirmed in another study using anti-IL-17A treatment (Supplementary Figure S5B). To summarize, multiple gene expression data of psoriatic patients before and after targeting the IL-17 pathway support our data that IL-17A regulates IL-19 expression in fibroblasts and keratinocytes.

\section{DISCUSSION}

In the present study, we showed that IL-10 neutralization enhanced skin inflammation, thickness and scaling in the IMQinduced psoriasis mouse model beyond day 5, via upregulation of the IL-17/IL-19 axis. IL-17A induced IL-19 and IL-24 expression in human dermal fibroblasts and epidermal keratinocytes, and IL-17A neutralization reduced the expression of both cytokines. Gene array expression data also show high expression of IL-17A, IL-19, IL-24 and proliferation marker Ki-67 in psoriatic skin lesions, and that anti-IL-17 therapy reduced their expression. In addition to keratinocytes, dermal fibroblasts, through interaction with immune cells and cytokines such as IL-17A and TNF in psoriasis, can be a major source of IL-19 and IL-24 that contribute to perpetuation of psoriatic symptoms such as keratinocyte proliferation and acanthosis.

In psoriasis, recombinant human IL-10 treatment has been demonstrated to improve psoriatic symptoms in clinical trials (20-23). Similarly, in the IMQ-induced psoriasis mouse model, a subset of IL-10-producing B cells was identified, and adoptive transfer of these IL-10-producing B cells reduced disease severity (24). In contrast, as shown by our and other groups, IL-10 neutralization or IL-10 deficiency induced persistent psoriasis-like inflammation after IMQ application (25). In psoriatic skin, macrophages and DC both can be producers as well as direct target cells of IL-10 (26). Frequency of Th17 cells can also be directly controlled by IL-10 and vice versa $(27,28)$. 

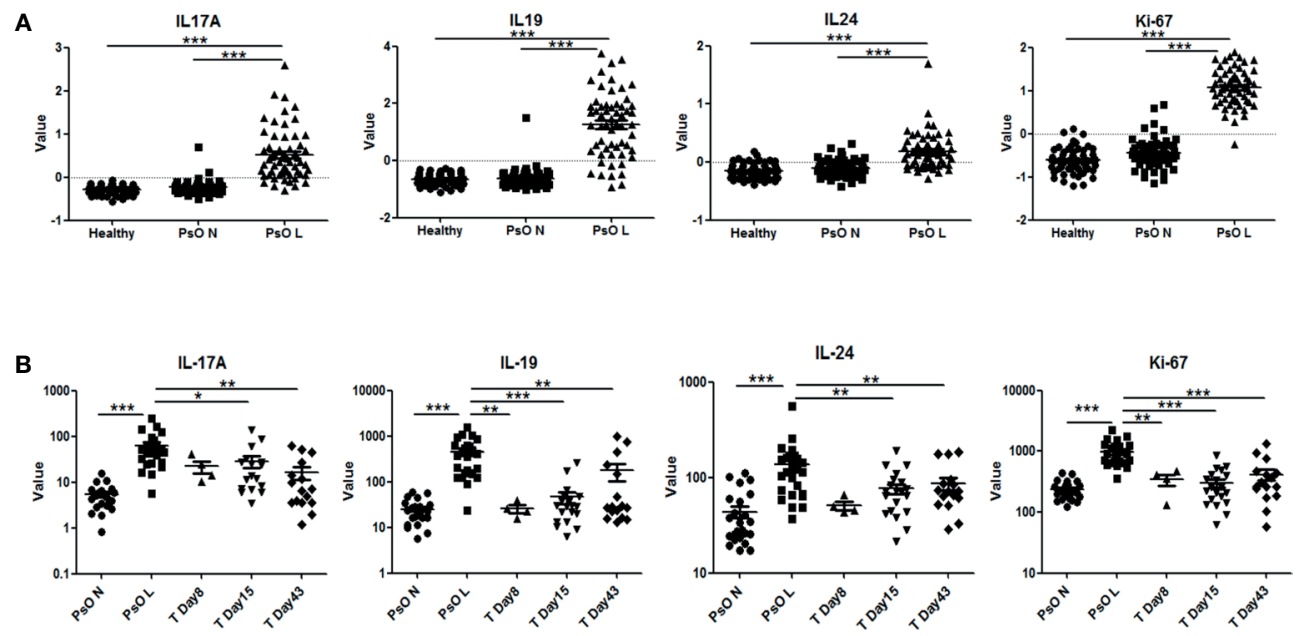

FIGURE 6 | Expression of human IL-17A, IL-19, IL-24 and Ki-67 in psoriatic lesions and the effects of anti-IL-17RA therapy. (A) Expression of IL-17A, IL-19, IL-24 and $\mathrm{Ki}-67$ in lesional and non-lesional skin biopsies from 58 psoriasis patients and in healthy skin biopsies from 64 normal controls in data set GSE13355. (B) Expression of IL-17A, IL-19, IL-24 and Ki-67 in lesional and non-lesional skin biopsies from 25 psoriasis patients before anti-IL-17RA treatment, 8,15 and 43 days after treatments in data set GSE53552. Data are shown as means \pm SEMs. ${ }^{*} P<0.05,{ }^{\star \star} P<0.01$, and ${ }^{\star \star \star} P<0.001$

IL-10 belongs to the cytokine family of IL-19, IL-20, IL-22 and IL-24 and are located in the same cluster on chromosome 1 $(29,30)$. Although IL-10 is the only anti-inflammatory cytokine in this family, simultaneous induction of IL-10 and the IL-20 subfamily has been observed in monocytes by stimulants (31). IL-10 production during inflammation acts as a natural counterbalance to limit the side effects of inflammation. Without this Yin-Yang dynamic equilibrium, inflammation will be skewed towards uncontrolled harmful diseases. For instance, in psoriasis, low levels of IL-10 have been reported in comparison to other inflammatory skin conditions, while contrarily, enhanced expression of IL-23/IL-17 pathway cytokines has been widely confirmed in psoriatic lesions $(9,32,33)$.

Overexpression of IL-19 and IL-24 has also been observed in psoriatic skin and both induce keratinocyte hyper-proliferation in a reconstituted human skin model (34-36), suggesting a pathogenic role in psoriasis. Myeloid cells are producers of both cytokines, and keratinocytes are also potential producers (29, 30, 37). Recently, IL-19 was suggested as an important mediator of the IL-23/IL-17 cascade in psoriasis, and IL-17Ainduced expression of IL-19 in keratinocytes amplifies keratinocyte responses via auto-paracrine regulation (14). Here we confirmed the induction of IL-19 by IL-17A in human keratinocytes and extended to show that IL-17A neutralization reduced IL-19 in human keratinocyte-T cell co-cultures (Supplementary Figure S4B). In addition, our study provided evidence that dermal fibroblasts also produced IL-19 and IL-24 in response to IL-1 $\beta$ and IL-17A, and, when co-cultured with activated memory $\mathrm{T}$ cells, fibroblasts produced significant protein levels of IL-19 (Figures 5A-C). Synergistic induction of IL-20 subfamily cytokines by IL- $1 \beta$ and IL-17A has also been observed in recent publications (38). Interestingly, in psoriatic fibroblast-T cell co-cultures, higher levels of IL-19 was observed in comparison to healthy fibroblast co-cultures, further supporting the contribution of psoriatic fibroblasts to local IL19 production. Compared to epidermal keratinocytes, dermal fibroblasts are positioned to encounter more frequently with inflammatory cells including $\mathrm{T}$ cells as most infiltrating $\mathrm{T}$ cells accumulate in the dermis (Supplementary Figure S3A). Therefore, in psoriasis, dermal fibroblasts could be an important local source of IL-20 subfamily cytokines and contribute to keratinocyte hyper-proliferation through a paracrine mechanism.

Discordant regulation of IL-20 in contrast to IL-19 and IL-24 has been found in our study. This could be due to different immune and resident cell types as potential cellular sources for these cytokines (30). Like skin fibroblasts and keratinocytes, other tissue cells such as endothelial cells and fibroblast-like synovial cells can also produce IL-20 subfamily cytokines (30, 39). Nevertheless, whether blocking the IL-20 subfamily cytokines will be viable options in psoriasis treatments still warrants further research.

In summary, we show that IL-10 regulates the expression of cytokines related to the IL-23/IL-17 axis via IL-19 and IL-24 influencing skin thickness and scaling. These data give further insight into the cytokine network in the stromal milieu of psoriasis plaques. Strategies to upregulate local or systemic production of IL-10 in patients with psoriasis could help increasing the effectiveness of current therapies.

\section{DATA AVAILABILITY STATEMENT}

The original contributions presented in the study are included in the article/Supplementary Material. Further inquiries can be directed to the corresponding author. 


\section{ETHICS STATEMENT}

The studies involving human participants were reviewed and approved by Medical Research Ethics Committee Erasmus MC. The patients/participants provided their written informed consent to participate in this study. The animal study was reviewed and approved by Erasmus MC Dutch Animal Ethics Committee (DEC).

\section{AUTHOR CONTRIBUTIONS}

XX contributed to the study design, performed experiments and wrote the manuscript. EF, PA, and A-MM performed experiments and revised the manuscript. PL and LB supported experiments and revised the manuscript. EP contributed to the study design and revised the manuscript. EL designed the study

\section{REFERENCES}

1. Nestle FO, Kaplan DH, Barker J. Psoriasis. N Engl J Med (2009) 361:496-509. doi: 10.1056/NEJMra0804595

2. Greb JE, Goldminz AM, Elder JT, Lebwohl MG, Gladman DD, Wu JJ, et al. Psoriasis. Nat Rev Dis Primers (2016) 2:16082. doi: 10.1038/nrdp.2016.82

3. Griffiths CE, Barker JN. Pathogenesis and Clinical Features of Psoriasis. Lancet (2007) 370:263-71. doi: 10.1016/S0140-6736(07)61128-3

4. Nickoloff BJ. The Cytokine Network in Psoriasis. Arch Dermatol (1991) 127:871-84. doi: 10.1001/archderm.1991.01680050115015

5. Johansen C, Usher PA, Kjellerup RB, Lundsgaard D, Iversen L, Kragballe K. Characterization of the Interleukin-17 Isoforms and Receptors in Lesional Psoriatic Skin. Br J Dermatol (2009) 160:319-24. doi: 10.1111/j.13652133.2008.08902.x

6. Soderstrom C, Berstein G, Zhang W, Valdez H, Fitz L, Kuhn M, et al. UltraSensitive Measurement of IL-17A and IL-17F in Psoriasis Patient Serum and Skin. AAPS J (2017) 19:1218-22. doi: 10.1208/s12248-017-0094-4

7. Lowes MA, Kikuchi T, Fuentes-Duculan J, Cardinale I, Zaba LC, Haider AS, et al. Psoriasis Vulgaris Lesions Contain Discrete Populations of Th1 and Th17 T Cells. J Invest Dermatol (2008) 128:1207-11. doi: 10.1038/ sj.jid.5701213

8. Ortega C, Fernández -S, Carrillo JM, Romero P, Molina IJ, Moreno JC, et al. IL-17-Producing CD8+ T Lymphocytes From Psoriasis Skin Plaques Are Cytotoxic Effector Cells That Secrete Th17-Related Cytokines. J Leukoc Biol (2009) 86:435-43. doi: 10.1189/JLB.0109046

9. Cai Y, Shen X, Ding C, Qi C, Li K, Li X, et al. Pivotal Role of Dermal IL-17Producing $\gamma \delta$ T Cells in Skin Inflammation. Immunity (2011) 35:596-610. doi: 10.1016/j.immuni.2011.08.001

10. Krueger JG, Brunner PM. Interleukin-17 Alters the Biology of Many Cell Types Involved in the Genesis of Psoriasis, Systemic Inflammation and Associated Comorbidities. Exp Dermatol (2018) 27:115-23. doi: 10.1111/ exd.13467

11. Wang CQF, Akalu YT, Suarez-Farinas M, Gonzalez J, Mitsui H, Lowes MA, et al. IL-17 and TNF Synergistically Modulate Cytokine Expression While Suppressing Melanogenesis: Potential Relevance to Psoriasis. J Invest Dermatol (2013) 133:2741-52. doi: 10.1038/jid.2013.237

12. Hawkes JE, Yan BY, Chan TC, Krueger JG. Discovery of the IL-23/IL-17 Signaling Pathway and the Treatment of Psoriasis. J Immunol (2018) 201:1605-13. doi: 10.4049/jimmunol.1800013

13. Li HH, Lin YC, Chen PJ, Hsiao CH, Lee JY, Chen WC, et al. Interleukin-19 Upregulates Keratinocyte Growth Factor and is Associated With Psoriasis. $\mathrm{Br}$ J Dermatol (2005) 153(3):591-5. doi: 10.1111/j.1365-2133.2005.06665.x

14. Witte E, Kokolakis G, Witte K, Philipp S, Doecke WD, Babel N, et al. IL-19 is a Component of the Pathogenetic IL-23/IL-17 Cascade in Psoriasis. J Invest Dermatol (2014) 134:2757-67. doi: 10.1038/jid.2014.308 and revised the manuscript. All authors contributed to the article and approved the submitted version.

\section{FUNDING}

The current project is funded by the Departments of Rheumatology and Dermatology, Erasmus MC, University Medical Center Rotterdam. XX is supported by a scholarship under China State Scholarship Fund (CSC No.201406100056).

\section{SUPPLEMENTARY MATERIAL}

The Supplementary Material for this article can be found online at: https://www.frontiersin.org/articles/10.3389/fimmu.2021.719562/ full\#supplementary-material

15. Konrad RJ, Higgs RE, Rodgers GH, Ming W, Qian YW, Bivi N, et al. Assessment and Clinical Relevance of Serum IL-19 Levels in Psoriasis and Atopic Dermatitis Using a Sensitive and Specific Novel Immunoassay. Sci Rep (2019) 9:5211. doi: 10.1038/s41598-019-41609-Z

16. van der Fits L, Mourits S, Voerman JS, Kant M, Boon L, Laman JD, et al. Imiquimod-Induced Psoriasis-Like Skin Inflammation in Mice Is Mediated via the IL-23/IL-17 Axis. J Immunol (2009) 182:5836-45. doi: 10.4049/ jimmunol.0802999

17. Vinter H, Iversen L, Steiniche T, Kragballe K, Johansen C. Aldara-Induced Skin Inflammation: Studies of Patients With Psoriasis. Br J Dermatol (2015) 172:345-53. doi: 10.1111/bjd.13236

18. van Hamburg JP, Asmawidjaja PS, Davelaar N, Mus AM, Colin EM, Hazes JM, et al. Th17 Cells, But Not Th1 Cells, From Patients With Early Rheumatoid Arthritis are Potent Inducers of Matrix Metalloproteinases and Proinflammatory Cytokines Upon Synovial Fibroblast Interaction, Including Autocrine Interleukin-17A Production. Arthritis Rheum (2011) 63:73-83. doi: 10.1002/art.30093

19. Van Belle AB, de Heusch M, Lemaire MM, Hendrickx E, Warnier G, DunussiJoannopoulos K, et al. IL-22 Is Required for Imiquimod-Induced Psoriasiform Skin Inflammation in Mice. J Immunol (2012) 188:462-9. doi: 10.4049/ jimmunol.1102224

20. Asadullah K, Sterry W, Stephanek K, Jasulaitis D, Leupold M, Audring H, et al. IL-10 Is a Key Cytokine in Psoriasis. Proof of Principle by IL-10 Therapy: A New Therapeutic Approach. J Clin Invest (1998) 101:783-94. doi: 10.1172/ JCI1476

21. Friedrich M, Döcke WD, Klein A, Philipp S, Volk HD, Sterry W, et al. Immunomodulation by Interleukin-10 Therapy Decreases the Incidence of Relapse and Prolongs the Relapse-Free Interval in Psoriasis. J Invest Dermatol (2002) 118:672-7. doi: 10.1046/j.1523-1747.2002.01731.x

22. Reich K, Garbe C, Blaschke V, Maurer C, Middel P, Westphal G, et al. Response of Psoriasis to Interleukin-10 Is Associated With Suppression of Cutaneous Type 1 Inflammation, Downregulation of the Epidermal Interleukin-8/CXCR2 Pathway and Normalization of Keratinocyte Maturation. J Invest Dermatol (2001) 116:319-29. doi: 10.1046/j.1523-1747.2001.01248.x

23. McInnes IB, Illei GG, Danning CL, Yarboro CH, Crane M, Kuroiwa T, et al. IL-10 Improves Skin Disease and Modulates Endothelial Activation and Leukocyte Effector Function in Patients With Psoriatic Arthritis. J Immunol (2001) 167:4075-82. doi: 10.4049/jimmunol.167.7.4075

24. Yanaba K, Kamata M, Ishiura N, Shibata S, Asano Y, Tada Y, et al. Regulatory B Cells Suppress Imiquimod-Induced, Psoriasis-Like Skin Inflammation. J Leukoc Biol (2013) 94:563-73. doi: 10.1189/jlb.1112562

25. Jin SP, Koh SJ, Yu DA, Kim MW, Yun HT, Lee DH, et al. Imiquimod-Applied Interleukin-10 Deficient Mice Better Reflects Severe and Persistent Psoriasis With Systemic Inflammatory State. Exp Dermatol (2018) 27:43-9. doi: 10.1111/exd.13403 
26. Saraiva M, O'Garra A. The Regulation of IL-10 Production by Immune Cells. Nat Rev Immunol (2010) 10:170-81. doi: 10.1038/nri2711

27. Huber S, Gagliani N, Esplugues E, O'Connor W Jr, Huber FJ, Chaudhry A, et al. Th17 Cells Express Interleukin-10 Receptor and Are Controlled by Foxp3 ${ }^{-}$and Foxp3+ Regulatory CD4+ T Cells in an Interleukin-10Dependent Manner. Immunity (2011) 34:554-65. doi: 10.1016/j.immuni. 2011.01.020

28. Shimizu T, Kamata M, Fukaya S, Hayashi K, Fukuyasu A, Tanaka T, et al. Anti-IL-17A and IL-23p19 Antibodies But Not Anti-Tnfo Antibody Induce Expansion of Regulatory $\mathrm{T}$ Cells and Restoration of Their Suppressive Function in Imiquimod-Induced Psoriasiform Dermatitis. J Dermatol Sci (2019) 95:90-8. doi: 10.1016/j.jdermsci.2019.07.006

29. Rutz S, Wang X, Ouyang W. The IL-20 Subfamily of Cytokines-From Host Defence to Tissue Homeostasis. Nat Rev Immunol (2014) 14:783-95. doi: $10.1038 /$ nri3766

30. Sabat R. IL-10 Family of Cytokines. Cytokine Growth Factor Rev (2010) 21:315-24. doi: 10.1016/j.cytogfr.2010.11.001

31. Wolk K, Kunz S, Asadullah K, Sabat R. Cutting Edge: Immune Cells as Sources and Targets of the IL-10 Family Members? J Immunol (2002) 168:5397-402. doi: 10.4049/jimmunol.168.11.5397

32. Nickoloff BJ, Fivenson DP, Kunkel SL, Strieter RM, Turka LA. Keratinocyte Interleukin-10 Expression Is Upregulated in Tape-Stripped Skin, Poison Ivy Dermatitis, and Sezary Syndrome, But Not in Psoriatic Plaques. Clin Immunol Immunopathol (1994) 73:63-8. doi: 10.1006/clin.1994.1170

33. Mussi A, Bonifati C, Carducci M, Viola M, Tomaselli R, Sacerdoti G, et al. IL10 Levels are Decreased in Psoriatic Lesional Skin as Compared to the Psoriatic Lesion-Free and Normal Skin Suction Blister Fluids. J Biol Regul Homeost Agents (1994) 8:117-20.

34. Otkjaer K, Kragballe K, Funding AT, Clausen JT, Noerby PL, Steiniche T, et al. The Dynamics of Gene Expression of Interleukin-19 and Interleukin-20 and Their Receptors in Psoriasis. Br J Dermatol (2005) 153:911-8. doi: 10.1111/ j.1365-2133.2005.06800.x

35. Rømer J, Hasselager E, Nørby PL, Steiniche T, Thorn Clausen J, Kragballe K. Epidermal Overexpression of Interleukin-19 and -20 mRNA in Psoriatic Skin Disappears After Short-Term Treatment With Cyclosporine a or
Calcipotriol. J Invest Dermatol (2003) 121:1306-11. doi: 10.1111/j.15231747.2003.12626.x

36. Sa SM, Valdez PA, Wu J, Jung K, Zhong F, Hall L, et al. The Effects of IL-20 Subfamily Cytokines on Reconstituted Human Epidermis Suggest Potential Roles in Cutaneous Innate Defense and Pathogenic Adaptive Immunity in Psoriasis. J Immunol (2007) 178:2229-40. doi: 10.4049/jimmunol.178.4.2229

37. Yano S, Banno T, Walsh R, Blumenberg M. Transcriptional Responses of Human Epidermal Keratinocytes to Cytokine Interleukin-1. J Cell Physiol (2008) 214:1-13. doi: 10.1002/jcp.21300

38. Bertelsen T, Iversen L, Johansen C. I-Kappa-B-Zeta Regulates Interleukin17A/Tumor Necrosis Factor-Alpha Mediated Synergistic Induction of Interleukin-19 and Interleukin-20 in Humane Keratinocytes. Ann Dermatol (2021) 33:122-30. doi: 10.5021/ad.2021.33.2.122

39. Sakurai N, Kuroiwa T, Ikeuchi H, Hiramatsu N, Maeshima A, Kaneko Y, et al. Expression of IL-19 and Its Receptors in RA: Potential Role for Synovial Hyperplasia Formation. Rheumatol (Oxford) (2008) 47:815-20. doi: 10.1093/ rheumatology/ken061

Conflict of Interest: The authors declare that the research was conducted in the absence of any commercial or financial relationships that could be construed as a potential conflict of interest.

Publisher's Note: All claims expressed in this article are solely those of the authors and do not necessarily represent those of their affiliated organizations, or those of the publisher, the editors and the reviewers. Any product that may be evaluated in this article, or claim that may be made by its manufacturer, is not guaranteed or endorsed by the publisher.

Copyright (๑) $2021 \mathrm{Xu}$, Prens, Florencia, Leenen, Boon, Asmawidjaja, Mus and Lubberts. This is an open-access article distributed under the terms of the Creative Commons Attribution License (CC BY). The use, distribution or reproduction in other forums is permitted, provided the original author(s) and the copyright owner(s) are credited and that the original publication in this journal is cited, in accordance with accepted academic practice. No use, distribution or reproduction is permitted which does not comply with these terms. 